\title{
ESTUDIO DE UN NUEVO QUIMIOTERAPICO MERCURIAL
}

\author{
II ACTIVIDAD ANTIBACTERIANA \\ Drem. PLUTARCO NARANIO Y JULIA DE MOKENO

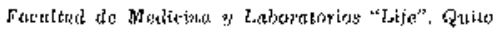

En la I parte de essta serie de trabajos ${ }^{1}$, ae describieron los efectos túxicos ded estearates sulfosuto (oetadeciltrimetilanonis) mercúrico (S-200 ${ }_{n}$ Cuлtuctín), substancia sintetizada bajo las hipristesis de que debia poseer propiedarles antibacteriarlas cotno sucede con los detergentes outiónícuss-4 y los comrpucstos orgánicos dell nascurify-i.

î́n el presente thabajo se diterminó la acividad antimicrobiana, frentc it gérmenes tanto Gram-pusitivos conu Gran-negativos. bl estudio se efoctuó comparadivamente con varius otros auimistorápicus. 'También se ensayó un posible afecto lisantc.

\section{MATHELALISS Y METODOS}

Para determinar cutintitativurmente el poder inhibitorio del desarrollo bacterierte se siguió el mótodo de la cilución sucesiva, utjlizando series de it) tubos, en los cue la droga en estudio: tho chloyćndose al 1:Z sucusivamente El medio de cultrvo empleado parra exta serice do ensayos, correspondió a la sì-

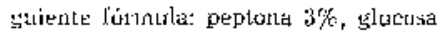

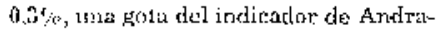
die (rojo fenol). E! pH se ajusstó a 7,4 $y$ ste eslerilizó a $1.18^{\circ} \mathrm{C}$, en alloclave.

F. "inóclolo" se prepuró agregatzdo - ll:0 mi, del mencionato medior de enlitro $0,5 \mathrm{~m}$ ! cle un cultivo de la bacteria en estutio, mantenida en inldas normal por 24 horas, is $37^{\prime \prime} \mathrm{C}$.

In los tubos se hizo previarasule de dilusión de la drorga en solución salina (solucion de NecL ul 0,9\%), quedanda in wolumen final de 0,5 wal. al eual se agrego $1,5 \mathrm{ml}$. dol inóculo. Se agitó bien y luergo se puso a incubar a $37^{\circ} \mathrm{C}$ : f:ir 18 a 24 horas.

La lestuxa de fos xestulados se hizo por el viraje del color del indicador de Andritde. En los tubos donde hubo desarrulto de bacterias el color rosado Anl medio viró a amariljo $y$, fácilmeute, pudo determinarse desde aué concen- 
Iración se prodajo la inhibición tatal.

A trás de la substamuia $\mathbf{3}-200$ ste ensayarn las siguientes: thiomerosai (Mertlixlate), rithofurazixa, ectrimide (Colievlón), Tecpol (dectergente de ori-

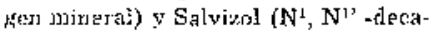
retilenc- $\mathrm{N}^{-1}, \quad \mathrm{~N}^{*}$ - dectanctilenc-bisatnino(ģuijalddine).

En tada serie experimental se estudió simultáneamente la actividad de

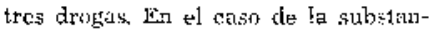
cia S.200, con algunos mickmorgunismos se rapitio el elusidro.

Lis microolganismos que sp ulilizaron pul'‘ exta investigación, en su mà yoúá se obtuvioron de paxientes que sufrían fa torrespondiente inleceíus. Con varias cepiss de Miserececeus pyogeties var. aureus (Sitilococo durado). se hicieron pruebas de sonsibjlirlad at los antibióticos.

Pora determinax si cI S-20) producía efecto lisante sc utilizó una sepa de Mizroceceus iysodeilstitus y se si-

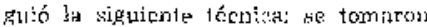
gél"nenes de lu cultivio de 24 horas, Ins que fuerus lavados put dos veves cott una solución tamponada correspousdiente a la siguiente fórmula: fosfat? :nonupotísico, 7,42 $\mathrm{gm}$; fosfato bisódino, $1,75 \overrightarrow{3}$ gitr.; clorum de sodin, 4,30 s

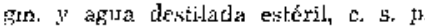

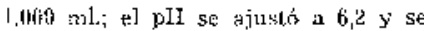

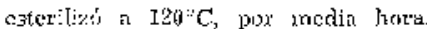
4 uejzo se preparó una suspensiún en el

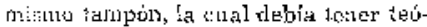
reannentr: la rrisma cantidat de bacte. viay, on rayón de que se usó una tuxbitior estúdsc, dsteminada en ol la!terilurituetro (Kleti - Summerson)

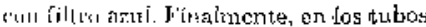

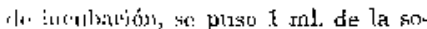

lución de la druga y 4 ml. dal inócule. Se incubó en ban̂o María a $37^{\circ} \mathrm{C}$, yor $\int 5$ minutos $y$ luego se velvio a "leer" la tubidez en el intocolorimeiro. Se utilizaron los testigos neresarios.

\section{RESULTADOS}

1) Actividad antibacteriana.—La tabla I presenta lus rescltiodos ubteni. dos en lat dicientes series de enzayos. r.tous puede noscrvarse, In sensibilidad de las valias cepos de M. pyogenes Vilt: aureus varió según cada una di etins, hibbiundo sido necesario concentrac:(1)ms mirt:mas desde $0,78 \mathrm{megm} / \mathrm{ml}$ linsia 12,5 mepmiml de la substancia $S .260$, para producir la tumpleta inhibistion de: deserrollo de este mieroorýtristrits. En Ia mayoría de los casos la ementración mínitrin efectiva fur: औa $0,78 \mathrm{mcgm} / \mathrm{mI}$

Én romparación con las otras substancias estudiarlas so observa qut ac requirieron, para obtener jgatal efectu, concenlreciones de thiamerosal $y$ de cetrimuide, muy semejantes a la de la subetincia s-2uk, pudiendo sonsidexarae jgual le potencia antisacteriana de csitas 3 zirogas sobre el MI. pyngcues sar. alijeis.

Site reçuirieron concentraciones 10 a 20 reces stperiorts die Selvizol y 50 a 100 veces unayores de Tecpol y ritru. tirazinel, sjenclo pues la potencia antibactsy:ann de exiacs druses mu infetjo: : lit de lis tros primertmente menciomadas.

Juit sensibilidad del ha. pyogenes var. aureus a astos antisépticos o quimiuterápicos, incluxive la arbstameis $\mathbf{5}-200$, 


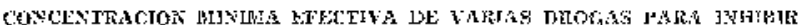

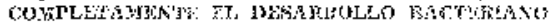

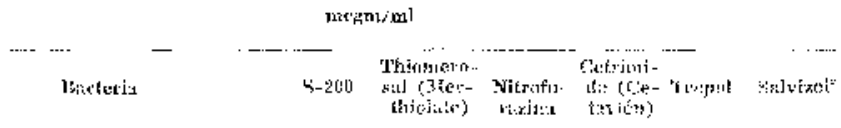

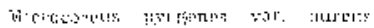

briscl:

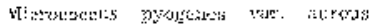

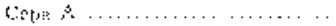

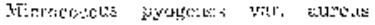

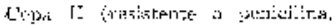

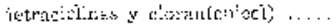

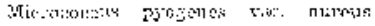

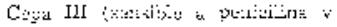

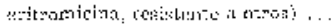

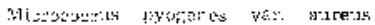

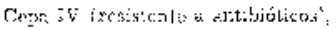

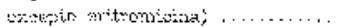

0.78

1.75

t. 2.2

L.2.

$3,1: 3$

1:.

(b,is

$4: 7 i s$

[j, Ê:\%

6.25

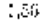

1),

1.54

is:

2.519

1). 78

$1,7,78$

0,162

?

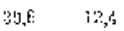

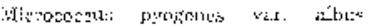

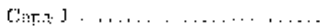

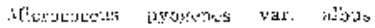

$3,1 \% \quad$ 1 $198 \quad$

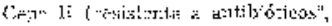

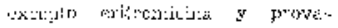

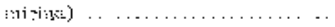

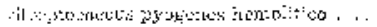

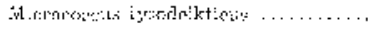

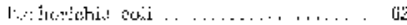

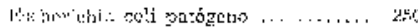

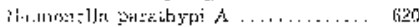

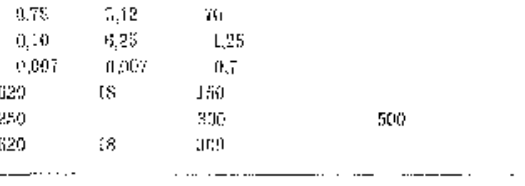

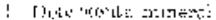

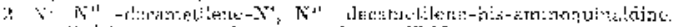

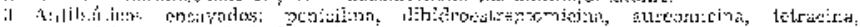

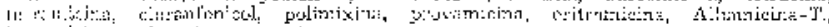

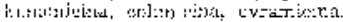

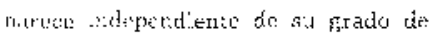

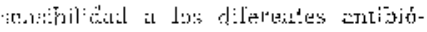
ticos.

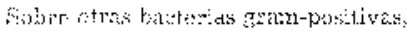
la anctiv:

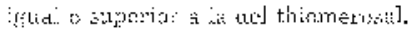

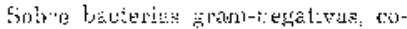

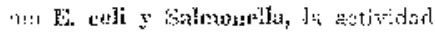

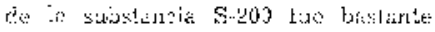

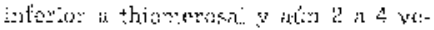

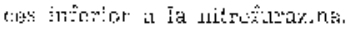

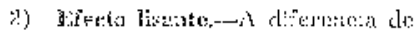

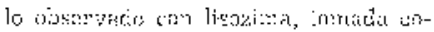

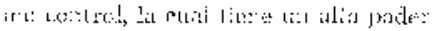

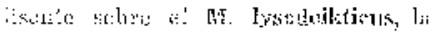

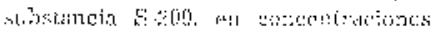


Jcerde 1:3.900, Jiasta 1:100; shes dermostró ninglina activirlad liswnte, I+at turbidex estrutdar (350 en el fotucolorimetro),

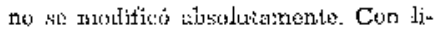

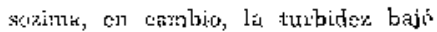
a lfio, cuando la coonecntraciún fue de

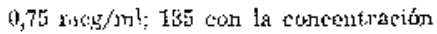
de 0,50 negrim y 240 con $\mathrm{I}_{2}$ conconłación de $0,2,5 \mathrm{mcr} / 1 \mathrm{ml}$.

\section{CONCLUSIONES}

1) Tso substancia S-iano pusee im alto puder antihacterianu, sientu muchri mayos kotre gérmenes gram-posin livos gale sobste grani-nerativos.

2) Lid activiclad antihacteriana che la sulystancia S-200, sohre gémenes Ciran-pusitixus ex igual a stperjor a la del thiomerosat y el cetritride y muy superior a la del soluvizol, el Teepol $y$ la riluofuxazima. En cambio subre frimernes (xt)ary-llegitivos su actividad es bessumte inferior a la klel thiometosal $y$ alin inferior a jutrofuresina.

3) La substancia S-200 no produce biogúr etecto lisatrte subre el M. Mysodeikticus.

\section{SUMMARY}

Antibacterial activity ot a mercuric cimpulod stearate sulphomale (octadecyiltrimethyl ammonitur) mercuric (S-200)-was studjed. It was found that S-200 prucluced a strong antibanterial effuct whith is more sichettive upon gram-positive microo:gaujisms the upon granl-3ogative gems.

The ietivity of S-200 on grani-positive beckeria was similar lo or higher ithan thiumerasal and cotrimide and stromely higher thin Salvizul, Teepol and nitroburazisu. On the othes band, aetivity on gram-ncgative germs was lower thas thiomerusal and ever nitrofurazine.

5-201) did nol produce any litic effect on M. lysodeikticus.

\section{BWETENCTAS DOBLIOGRATICAS}

1. NAFIANJO, P.. DF NARANJO, E. C HIDALC0, G.: Estuljo cle tu nuswo quinis-

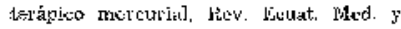
Cieni:. Hial. It: 7, 1964.

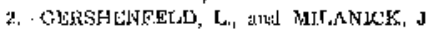

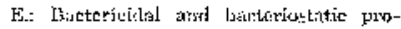
resties of surface terrion deptessants. Am. J. Pharan., 133: sidi, tokt.

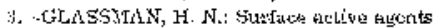

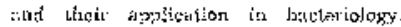
Bact. Iiev. 12: 105, ]d48.

4.-. MAVIS, II. L. (Bतitor), Synupozium, Me

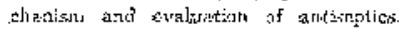
Ann, Now Yo!k deari. Se., 5iat 1, 3950.

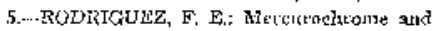

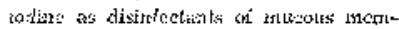
brine of moulth, J, A.7. H. A. 91; 708, 1028.

d.-OFT'JNGLN, w. E, von, CALHODr, 0 .

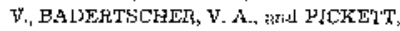

R. L.: Compartirese stedios on rnercurochrome and otinat andiseptics. J. Ars. MT. A. Ny: $127,1932$.

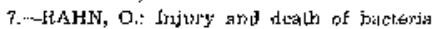
by chersictionents, Biodynomiea, Norแ1:andy, M.1., 1945. 